\title{
Triamcinolone acetonide combined with aminoguanidine inhibits inflammation and oxidative stress, improves vascular endothelial and retinal function and reduces VEGF expression in diabetic retinopathy patients
}

\author{
$\mathrm{KAI} \mathrm{XU}^{1}$, HANLIANG QIAN ${ }^{2}$ and MEIBO $\mathrm{ZOU}^{2}$ \\ ${ }^{1}$ Department of Ophthalmology, Taizhou Second People's Hospital Affiliated to Yangzhou University, \\ Taizhou, Zhejiang 225500; ${ }^{2}$ Department of Ophthalmology, \\ Affiliated Hospital of Jiangsu University, Zhenjiang, Jiangsu 212001, P.R. China
}

Received November 13, 2019; Accepted January 9, 2020

DOI: $10.3892 /$ etm.2020.8478

\begin{abstract}
Effects of triamcinolone acetonide (TA) combined with aminoguanidine (AG) on vascular endothelial function, vascular endothelial growth factor (VEGF) expression and retinal function in diabetic retinopathy (DR) patients were studied. A total of 100 patients with DR admitted to the Taizhou Second People's Hospital Affiliated to Yangzhou University from January 2016 to December 2018 were collected and randomly divided into observation group $(n=50)$ and control group $(n=50)$. The control group was only treated with TA (4 mg), while the observation group was additionally treated with AG $(50 \mathrm{mg} / \mathrm{kg})$. The therapeutic effect and adverse reactions were observed, and the changes in blood glucose (GLU), blood lipid, serum inflammatory cytokines and oxidative stress indexes before and after treatment were detected. Moreover, the serum VEGF level was examined, the central macular thickness (CMT), retinal neovascularization (RNV) leakage area and best corrected visual acuity (BCVA) of retinopathy were observed, and the vascular endothelial function indexes such as nitric oxide (NO), endothelin-1 (ET-1) and intercellular adhesion molecule-1 (ICAM-1) were detected. The therapeutic effect in the observation group was significantly better than that in control group $(\mathrm{P}<0.05)$. In observation group after treatment, the levels of GLU, total cholesterol (TC), triglyceride (TG), interleukin-6 (IL-6), tumor necrosis factor- $\alpha$ (TNF- $\alpha$ ), myeloperoxidase (MPO), malondialdehyde (MDA) and catalase (CAT) in the serum, VEGF, CMT and
\end{abstract}

Correspondence to: Dr Hanliang Qian, Department of Ophthalmology, Affiliated Hospital of Jiangsu University, 438 Jiefang Road, Zhenjiang, Jiangsu 212001, P.R. China

E-mail: lianguai19940911@163.com

Key words: triamcinolone acetonide, aminoguanidine, diabetic retinopathy patients, inflammation, oxidative stress, vascular endothelial function, VEGF, retinal function
RNV, as well as NO, ET-1 and ICAM-1 were remarkably decreased $(\mathrm{P}<0.05)$, while those of superoxide dismutase (SOD) and BCVA were evidently increased $(\mathrm{P}<0.05)$. The changes were more significant at 3 months after treatment than those at 1 month. TA combined with AG can inhibit inflammation and oxidative stress, improve vascular endothelial function and retinal function and reduce VEGF expression in DR patients. The overall effect of the combined therapy is good, and its application is worth popularizing.

\section{Introduction}

Diabetes is one of the most common chronic metabolic disease in the world caused by multiple factors and has become one of the diseases with a high mortality rate globally. Patients with diabetes take drugs throughout their lives, which poses long-term economic pressure (1). Besides, diabetes has many complications threatening the vision. Cataract occurs earlier in diabetic patients. In many developed countries, diabetes is the major cause of non-invasive amputation, blindness and visual impairment of adults, and diabetic patients are more prone to suffering from glaucoma (2). Diabetes retinopathy (DR), a kind of retinal disease, is the main cause of blindness in developed countries. Its pathology is complex and affects the nerves and vascular components of the retina $(3,4)$. DR is featured with neurological dysfunction and the destruction of the retinal vascular system. Vascular complications are crucial factors for the progression of the disease. Retinal neuronal dysfunction arises in DR in the early stage and may even occur before vascular rupture $(5,6)$. Early neurological dysfunction is proved to be an abnormal electroretinogram response in diabetic rats and humans prior to any visible vascular injury (7). Additionally, decreased functional hyperemia is one of the earliest retinal changes in diabetic patients that are observed (8). The hyperglycemia activation of metabolic and biochemical pathways leads to irreversible changes, which are the adverse results of DR. DR is a continuously dependent disease that develops in stages. The incidence rate of DR is relatively low in the first few years of diabetes onset, but it 
will increase significantly after the onset of diabetes for many years. DR is the leading cause of acquired blindness in adults. If microvascular damage in the retina and vascular swelling and exudation are not prevented, new blood vessels will begin to grow, ultimately causing retinal detachment $(9,10)$. Inflammation is regarded as the key driving factor of DR pathophysiology. Raising the expression of pro-inflammatory cytokines, such as tumor necrosis factor- $\alpha$ (TNF- $\alpha)$, will further promote the occurrence of retinopathy in diabetic rats (11). Extensive attention has been paid to the function of oxidative stress in DR. Edaravone treatment for DR reduces the concentration of oxygen free radicals and suppresses delayed neuronal death (12).

Aminoguanidine (AG), a specific nitric oxide synthase (NOS) inhibitor, has been proven to slow down the progression of DR in human and animal diabetes models (13). These beneficial roles of AG are largely attributed to the repression on the formation of advanced glycation end products (AGEs). However, the results of multiple studies showed that AG may also function through inhibiting iNOS, especially in the early stage of the disease (14). Intravitreal triamcinolone acetonide (TA) can effectively eliminate macular edema and improve vision, especially for DR that cannot b(160e effectively treated by laser therapy, and has been widely applied in recent years. A study has revealed that TA can alleviate macular edema, prevent the aggravation of macular edema secondary to PRP, and facilitate the improvement of retinopathy (15).

In this study, the effects of TA combined with AG on vascular endothelial function, vascular endothelial growth factor (VEGF) expression and retinal function in DR patients were observed. DR patients were admitted and treated. The therapeutic effect and adverse reactions were observed, the changes in serum inflammatory cytokines and oxidative stress factors before treatment were detected, the level of serum VEGF was measured, and central macular thickness (CMT), retinal neovascularization ( $\mathrm{RNV})$, best corrected visual acuity (BCVA), nitric oxide (NO), endothelin-1 (ET-1), intercellular adhesion molecule-1 (ICAM-1) and other vascular endothelial function indexes were observed, to confirm that TA combined with AG could inhibit inflammation and oxidative stress and improve vascular endothelial function and retinal function in DR patients.

\section{Patients and methods}

Clinical data. The clinical research protocol was approved by the Ethics Committee of the Taizhou Second People's Hospital Affiliated to Yangzhou University (Taizhou, China). Signed informed consents were obtained from the patients and/or guardians. One hundred patients with DR admitted to the hospital from January 2016 to December 2018 were selected as research subjects. The patients were enrolled after signing an informed consent. They were divided into the observation group $(n=50)$ and the control group $(n=50)$ using random control method and informed of the condition of this study. Inclusion criteria: Patients with a history of diabetes, reduced vision in different degrees and different degrees of retinopathy shown in retinal fundus examination. Exclusion criteria: patients taking glucocorticoid drugs, those with severe organ dysfunction, those who could not receive intravenous injection, or those with gestational diabetes, hepatic and renal insufficiency, ketoacidosis, acute or chronic infection or immune system diseases. All clinical specimens in this experiment were collected with the consent of the patients and their family members according to the Helsinki Declaration. The specific clinical data collected on admission included age, sex, weight, course of disease and DR stage (Table I). No significant differences in the general data were found between the two groups of patients.

Treatment methods. All patients were treated with levofloxacin eye water in drops (1-2 drops/time and 4 drops/d) on the affected eye (until the water covered the whole eyeball) for 3 days before treatment, followed by conventional treatment for 3 consecutive days. After that, the patients in the control group underwent treatment with TA $(4 \mathrm{mg})$, while those in the observation group were treated with $\mathrm{AG}(50 \mathrm{mg} / \mathrm{kg})$ in addition. The specific injection method is as follows: Local anesthesia was carried out on the affected eye. After disinfection, the eyelid opener was applied to open the eyelid of the patients. Then the needle was inserted into the flat part of the corneal limbus of the patients' affected eye at $\sim 4 \mathrm{~mm}$. The direction of needle insertion was toward the center of the eye, with the depth of $\sim 4 \mathrm{~mm}$. The puncture site was ensured in the vitreous body. After withdrawing the needle, the needle site was pressed using cotton swabs. According to medical advice, the patients lied in the supine position for $1-2 \mathrm{~h}$ after the injection to ensure that the drug completely reached the posterior polar retina. They were treated once a day for 12 weeks.

Observation of therapeutic effect. Evaluation results of the therapeutic effect via optical coherence tomography (OCT) after treatment was divided into three types according to whether the morphology and edematous height were reduced, i.e. returning to normal, obvious improvement (the edematous height was reduced by more than $100 \mu \mathrm{m}$ compared with that before treatment) and no improvement (the edematous height was unchanged or increased compared with that before treatment).

Observation of adverse reactions. The complications of the two groups of patients after treatment were observed and recorded. The specially-assigned medical staff recorded the complications of anterior chamber inflammation, corneal edema, ocular hypertension and macular for the two groups of patients, respectively, counted the specific types of complications and made detailed records. Finally, the postoperative complications of the two groups of patients were summarized.

Detection of blood glucose (GLU), triglyceride (TG) and total cholesterol (TC). The biochemical indexes such as blood GLU and lipid will change in patients with diabetes. The occurrence and development of the disease can be indicated through the detection of the changes in the indexes. In the morning, $5 \mathrm{ml}$ of fasting peripheral venous blood of the patients was extracted and placed in an Eppendorf (EP) tube containing anticoagulant ethylenediaminetetraacetic acid (EDTA). The blood was centrifuged at $3,500 \mathrm{x} \mathrm{g}$ at room temperature for $10 \mathrm{~min}$, and the supernatant was collected to detect the changes in GLU, 
Table I. Clinical data.

\begin{tabular}{lcc}
\hline Indicator & $\begin{array}{c}\text { Control } \\
\text { group }\end{array}$ & $\begin{array}{c}\text { Observation } \\
\text { group }\end{array}$ \\
\hline Sample (n) & 50 & 50 \\
Male patient (n) & 30 & 29 \\
Average age (years) & $48 \pm 15$ & $49 \pm 16$ \\
Average body weight (kg) & $52 \pm 10.5$ & $54 \pm 11.5$ \\
Course of disease (years) & $3.5 \pm 1.5$ & $4.0 \pm 0.9$ \\
DR in stage III & 22 & 23 \\
DR in stage IV & 20 & 21 \\
DR in stage V & 8 & 6 \\
\hline
\end{tabular}

DR, diabetic retinopathy.

TG, TC and other indexes, so as to provide an important theoretical reference for early treatment.

Detection of serum inflammatory factors via enzyme-linked immunosorbent assay (ELISA). A total of $5 \mathrm{ml}$ of venous blood was extracted from the arm, placed in the EP tube containing anticoagulant and centrifuged at 2,500 $\mathrm{x} g$ at room temperature for $15 \mathrm{~min}$. Next, the supernatant was collected for the detection of serum inflammatory factors [interleukin-6 (IL-6), myeloperoxidase (MPO) and TNF- $\alpha$ ]. The specific operation was in accordance with the instructions of ELISA kit (Nanjing Jiancheng Bioengineering Institute). Then, the absorbance in each group was examined under a microplate reader.

Examination of serum oxidative stress indicators via ELISA. A total of $5 \mathrm{ml}$ of venous blood was extracted from the arm, placed in the EP tube containing anticoagulant and centrifuged at $2,500 \mathrm{x} \mathrm{g}$ at room temperature for $15 \mathrm{~min}$. Thereafter, the supernatant was collected for the detection of serum oxidative stress indicators [malondialdehyde (MDA), superoxide dismutase (SOD) and catalase (CAT)]. The specific operation was in line with the instructions of ELISA kit. Then, the absorbance in each group was measured by a microplate reader.

Detection of VEGF, NO, ET-1 and ICAM-1 in serum. A total of $5 \mathrm{ml}$ of venous blood was taken from patients and placed in the EP tube containing anticoagulant, followed by centrifugation at $2,500 \mathrm{x} \mathrm{g}$ at room temperature for $15 \mathrm{~min}$. The supernatant was collected to examine specific changes in the serum levels of VEGF, ET-1 and ICAM-1 using the kit. NO was determined by nitrate reductase colorimetry using a kit (Shanghai Hufeng Biotechnology Co., Ltd.). The specific operation followed the instructions of the kit. The absorbance in each group was examined under the microplate reader.

Observation of CMT, RNV and BCVA. OCT system (Zeiss, type: OCT3000OI-STD) was used to observe the CMT and RNV before and after treatment. The specific operation was performed by specially-assigned personnel according to the instrument instructions. The BCVA before and after treatment was observed on the basis of the international Visual Acuity Chart (version 2.0).

Statistical analysis. All the data originally recorded in the study were processed SPSS 20.0 (IBM Corp.) analysis software and compared in multiple ways. The percentage was tested using $\chi^{2}$ test. The experimental results were expressed as mean \pm standard deviation (mean $\pm \mathrm{SD}$ ). $\mathrm{P}<0.05$ indicates that the difference is statistically significant. The histograms were plotted using GraphPad Prism 6.0 (GraphPad Software)

\section{Results}

Observation results of the therapeutic effect in the two groups of patients. As shown in Table II, the total effective rate was $96 \%$ in the observation group and $74 \%$ in the control group, with a statistically significant difference $(\mathrm{P}<0.05)$.

Observation results of adverse reactions. The control group had 17 cases of complications, mainly including anterior chamber inflammation, corneal edema, ocular hypertension and macula, with the total adverse reaction rate of $34 \%$, while the observation group had only 4 cases of complications, with the total adverse reaction rate of $8 \%$, displaying a statistically significant difference $(\mathrm{P}<0.05)$ (Table III). Thus, TA combined with AG exerts an obvious effect on DR patients, with fewer adverse reactions and complications.

Detection results of GLU, TG and total TC. As shown in Table IV, GLU, TG and TC in the two groups were decreased after treatment, but the serum levels of TG, TC and GLU in the observation group were notably decreased compared with those in the control group $(\mathrm{P}<0.05)$, and the variation amplitude at 3 months after treatment was more evident than that at 1 month after treatment, indicating that the hyperlipemia indexes in DR patients will be clearly improved after treatment with TA combined with AG.

Detection results of serum inflammatory factors. Before treatment, there were no statistically significant differences in the levels of IL-6, MPO and TNF- $\alpha$ between the two groups of patients $(\mathrm{P}>0.05)$. After treatment, the levels of IL-6, MPO and TNF- $\alpha$ in the two groups of patients were remarkably lower than those before treatment, and the variation degree at 3 months after treatment was more obvious than that at 1 month after treatment. The levels in the observation group were significantly lower than those in the control group $(\mathrm{P}<0.05)($ Table V).

ELISA results of serum oxidative stress indicators. The examination results of oxidative stress indexes (SOD, MDA and CAT) are shown in Table VI. It was found that after treatment, the levels of MDA and CAT in the observation group declined markedly $(\mathrm{P}<0.05)$, the SOD level was evidently raised $(\mathrm{P}<0.05)$. The amplitude of variation at 3 months after treatment was larger than that at 1 month after treatment, and it was also larger in the observation group than that in the control group. 
Table II. Observation results of the therapeutic effect.

\begin{tabular}{lcccc}
\hline Groups & Returning to normal & Obvious improvement & No improvement & Total effective rate (\%) \\
\hline Control & 17 & 25 & 8 & 74 \\
Observation & $25^{\mathrm{a}}$ & $23^{\mathrm{a}}$ & $2^{\mathrm{a}}$ & $96^{\mathrm{a}}$ \\
\hline
\end{tabular}

In terms of the therapeutic effect, the total effective rate is $96 \%$ in observation group and $74 \%$ in control group, showing a statistically significant difference $(\mathrm{P}<0.05)$. ${ }^{\mathrm{a}} \mathrm{P}<0.05$ vs. control group.

Table III. Adverse reactions.

\begin{tabular}{|c|c|c|c|c|c|}
\hline Groups & $\begin{array}{l}\text { Inflammation of } \\
\text { anterior chamber }\end{array}$ & $\begin{array}{l}\text { Corneal } \\
\text { edema }\end{array}$ & $\begin{array}{l}\text { Intraocular } \\
\text { hypertension }\end{array}$ & Macula & $\begin{array}{c}\text { Total adverse } \\
\text { reaction rate }(\%)\end{array}$ \\
\hline Control & 3 & 2 & 5 & 7 & 34 \\
\hline Observation & $1^{\mathrm{a}}$ & $1^{\mathrm{a}}$ & $1^{\mathrm{a}}$ & $1^{\mathrm{a}}$ & $8^{a}$ \\
\hline
\end{tabular}

Control group had 17 cases of complications and the observation had 4 cases of complications, with a statistically significant difference $(\mathrm{P}<0.05)$. ${ }^{\mathrm{a}} \mathrm{P}<0.05$ vs. control group.

Table IV. Detection results of blood GLU, TG and TC.

\begin{tabular}{lccc}
\hline Group & TC $(\mathrm{mmol} / \mathrm{l})$ & TG $(\mathrm{mmol} / \mathrm{l})$ & GLU (mmol/l) \\
\hline Control group & & & $14.4 \pm 1.7$ \\
Before treatment & $5.5 \pm 0.6$ & $2.5 \pm 0.06$ & $9.9 \pm 1.2^{\mathrm{a}}$ \\
1 month after treatment & $3.9 \pm 0.8^{\mathrm{a}}$ & $1.9 \pm 0.04^{\mathrm{a}}$ & $5.5 \pm 1.5^{\mathrm{a}}$ \\
3 months after treatment & $2.8 \pm 0.1^{\mathrm{a}}$ & $1.2 \pm 0.09^{\mathrm{a}}$ & $15.0 \pm 4.7$ \\
Observation group & & & $6.7 \pm 2.6^{\mathrm{a}, \mathrm{b}}$ \\
Before treatment & $5.7 \pm 0.5$ & $1.3 \pm 0.04^{\mathrm{a}, \mathrm{b}}$ & $3.1 \pm 1.3^{\mathrm{a}, \mathrm{b}}$ \\
1 month after treatment & $2.3 \pm 0.4^{\mathrm{a}, \mathrm{b}}$ & $0.8 \pm 0.02^{\mathrm{a}, \mathrm{b}}$ & \\
3 months after treatment & $1.2 \pm 0.3^{\mathrm{a}, \mathrm{b}}$ & \\
\hline
\end{tabular}

GLU, TG and TC in the two groups decreased after treatment, but the serum levels of TG, TC and GLU in the observation group were notably reduced compared with those in the control group $(\mathrm{P}<0.05)$, and the variation amplitude at 3 months after treatment was more obvious than that at 1 month after treatment. ${ }^{a} \mathrm{P}<0.05$ vs. before treatment; ${ }^{\mathrm{b}} \mathrm{P}<0.05$ vs. control group in the same period. TG, triglyceride; TC, total cholesterol; GLU, changes in blood glucose.

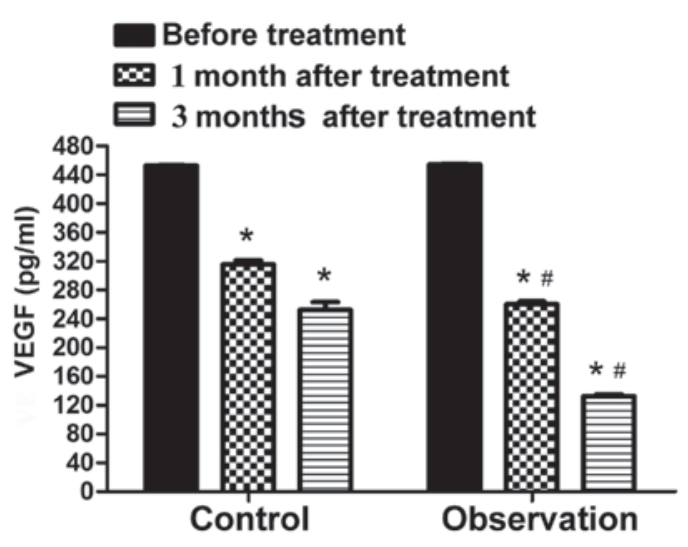

Figure 1. Changes in the VEGF level. After treatment, the VEGF level in the two groups of patients is notably lower than that before treatment, and it is markedly lower in observation than that in control group $(\mathrm{P}<0.05)$. ${ }^{*} \mathrm{P}<0.05$ vs. before treatment; ${ }^{\text {}} \mathrm{P}<0.05$ vs. control group in the same period. VEGF, vascular endothelial growth factor.
Serum VEGF expression. Fig. 1 shows that before treatment, there was no significant difference in the VEGF level between the two groups of patients $(P>0.05)$. After treatment, the VEGF level in the two groups of patients was notably lower than that before treatment, and it was markedly lower in the observation than that in the control group $(\mathrm{P}<0.05)$.

Examination results of vascular endothelial function indexes. Before treatment, the differences in the levels of NO, ET-1 and ICAM-1 were not statistically significant between the two groups of patients $(\mathrm{P}>0.05)$. After treatment, the levels of NO, ET-1 and ICAM-1 in the two groups of patients were remarkably lower than those before treatment, and the decrease at 3 months after treatment were more obvious than those at 1 month after treatment. The levels in the observation group were evidently lower than those in the control group $(\mathrm{P}<0.05)$ (Table VII). 
Table V. Serum levels of IL-6, MPO and TNF- $\alpha$.

\begin{tabular}{|c|c|c|c|}
\hline Groups & IL-6 (pg/ml) & $\mathrm{TNF}-\alpha(\mathrm{fmol} / \mathrm{ml})$ & MPO (ng/ml) \\
\hline \multicolumn{4}{|l|}{ Control } \\
\hline Before treatment & $69.5 \pm 2.6$ & $49.4 \pm 2.8$ & $218.5 \pm 1.8$ \\
\hline 1 month after treatment & $46.7 \pm 2.4^{\mathrm{a}}$ & $34.1 \pm 2.1^{\mathrm{a}}$ & $155.9 \pm 1.7^{\mathrm{a}}$ \\
\hline 3 months after treatment & $35.5 \pm 2.1^{\mathrm{a}}$ & $28.6 \pm 2.5^{\mathrm{a}}$ & $103.4 \pm 1.1^{\mathrm{a}}$ \\
\hline \multicolumn{4}{|l|}{ Observation } \\
\hline Before treatment & $69.1 \pm 1.3$ & $51.4 \pm 1.9$ & $217.9 \pm 4.7$ \\
\hline 1 month after treatment & $34.6 \pm 2.4^{\mathrm{a}, \mathrm{b}}$ & $26.7 \pm 1.1^{\mathrm{a}, \mathrm{b}}$ & $119.8 \pm 2.6^{\mathrm{a}, \mathrm{b}}$ \\
\hline 3 months after treatment & $23.1 \pm 2.5^{\mathrm{a}, \mathrm{b}}$ & $19.5 \pm 1.6^{\mathrm{a}, \mathrm{b}}$ & $89.6 \pm 2.4^{\mathrm{a}, \mathrm{b}}$ \\
\hline
\end{tabular}

Table VI. Serum oxidative stress indicators.

\begin{tabular}{|c|c|c|c|}
\hline Groups & CAT (IU/ml) & $\mathrm{MDA}(\mathrm{mmol} / \mathrm{g})$ & $\operatorname{SOD}(\mu / \mathrm{mg})$ \\
\hline \multicolumn{4}{|l|}{ Control } \\
\hline Before treatment & $30.5 \pm 2.1$ & $12.4 \pm 1.0$ & $15.5 \pm 1.4$ \\
\hline 1 month after treatment & $20.1 \pm 2.4^{\mathrm{a}}$ & $7.1 \pm 1.1^{\mathrm{a}}$ & $22.9 \pm 1.8^{\mathrm{a}}$ \\
\hline 3 months after treatment & $15.6 \pm 2.0^{\mathrm{a}}$ & $5.0 \pm 1.4^{\mathrm{a}}$ & $33.5 \pm 0.8^{\mathrm{a}}$ \\
\hline \multicolumn{4}{|l|}{ Observation } \\
\hline Before treatment & $31.1 \pm 1.2$ & $11.9 \pm 0.9$ & $16.1 \pm 1.3$ \\
\hline 1 month after treatment & $16.4 \pm 2.0^{\mathrm{a}, \mathrm{b}}$ & $5.5 \pm 0.8^{\mathrm{a}, \mathrm{b}}$ & $30.4 \pm 1.6^{\mathrm{a}, \mathrm{b}}$ \\
\hline 3 months after treatment & $9.8 \pm 2.1^{\mathrm{a}, \mathrm{b}}$ & $2.3 \pm 0.1^{\mathrm{a}, \mathrm{b}}$ & $43.1 \pm 1.7^{a, b}$ \\
\hline
\end{tabular}

Oxidative stress indicators: After treatment, the levels of MDA and CAT in the observation group decline markedly $(\mathrm{P}<0.05)$, and the SOD level is markedly raised $(\mathrm{P}<0.05)$. The amplitude of variation at 3 months after treatment is larger than that at 1 month after treatment, and it is also larger in observation group than that in control group. ${ }^{\mathrm{a}} \mathrm{P}<0.05 \mathrm{vs}$. before treatment; ${ }^{\mathrm{b}} \mathrm{P}<0.05 \mathrm{vs}$. control group in the same period. CAT, catalase; MDA, malondialdehyde; SOD, superoxide dismutase.

Examination results of CMT, RNV and BCVA. As shown in Table VIII, statistically significant differences in the levels of CMT, RNV and BCVA were detected prior to treatment between the two groups of patients. After treatment, the levels of CMT and RNV were remarkably decreased while BCVA level was markedly increased. The amplitude of variation at 3 months after treatment was more significant than that at 1 month after treatment, and it was also more obvious in the observation group than that in control group $(\mathrm{P}<0.05)$.

\section{Discussion}

DR is a serious complication related to diabetes (16). During diabetes, the age of retinal pericytes gradually increases, producing adverse effects on cell function and survival. Early DR is featured with loss of retinal pericytes that may result in an increase in the ratio of endothelial cells to pericytes (17). In vivo, hyperglycemia-induced AGEs are deposited in retinal vessels, which play crucial roles in the occurrence and development of DR. Age also causes the loss of retinal pericytes in healthy rats. Inhibition of cytotoxicity mediated by age has been adopted as a treatment regimen to prevent diabetic complications (18). In this study, DR patients were enrolled and treated, the therapeutic effect, adverse reactions, CMT, RNV and BCVA were observed, and NO, ET-1, ICAM-1 and other vascular endothelial function indexes were detected. The total effective rate was $96 \%$ in the observation group and $74 \%$ in the control group. The control group had 17 cases of complications, mainly including anterior chamber inflammation, corneal edema, ocular hypertension and macula, with the total adverse reaction rate of $34 \%$, while observation group had 4 cases of complications, with the total adverse reaction rate of $8 \%$, displaying a statistically significant difference. Thus, TA combined with AG exerts an obvious effect on DR patients, with fewer adverse reactions and complications. In addition, it was discovered that GLU, TG and TC in the two groups were decreased after treatment, but the serum levels of TG, TC and GLU in the observation group were notably 
Table VII. Levels of NO, ET-1 and ICAM-1.

\begin{tabular}{lccc}
\hline Groups & $\mathrm{NO}(\mu \mathrm{mol} / \mathrm{l})$ & ET-1 $(\mathrm{ng} / \mathrm{l})$ & ICAM-1 $(\mu \mathrm{g} / \mathrm{l})$ \\
\hline Control & & & $325.5 \pm 8.4$ \\
Before treatment & $82.4 \pm 4.7$ & $25.4 \pm 1.7$ & $271.9 \pm 9.8^{\mathrm{a}}$ \\
1 month after treatment & $55.1 \pm 5.1^{\mathrm{a}}$ & $20.1 \pm 1.2^{\mathrm{a}}$ & $230.7 \pm 7.8^{\mathrm{a}}$ \\
3 months after treatment & $44.8 \pm 5.5^{\mathrm{a}}$ & $15.4 \pm 1.5^{\mathrm{a}}$ & $326.1 \pm 10.3$ \\
Observation & & & $26.9 \pm 1.3$ \\
Before treatment & $82.9 \pm 4.9$ & $14.6 \pm 1.1^{\mathrm{a}, \mathrm{b}}$ & $209.4 \pm 7.6^{\mathrm{a}, \mathrm{b}}$ \\
1 month after treatment & $46.5 \pm 3.8^{\mathrm{a}, \mathrm{b}}$ & $5.6 \pm 1.8^{\mathrm{a}, \mathrm{b}}$ & $150.5 \pm 7.3^{\mathrm{a}, \mathrm{b}}$ \\
3 months after treatment & $36.7 \pm 3.4^{\mathrm{a}, \mathrm{b}}$ & & \\
\hline
\end{tabular}

After treatment, the levels of NO, ET-1 and ICAM-1 in the two groups of patients are remarkably lower than those before treatment, and the decreases at 3 months after treatment are more obvious than those at 1 month after treatment. Besides, these levels in the observation group are evidently lower than those in the control group $(\mathrm{P}<0.05)$. ${ }^{\mathrm{a}} \mathrm{P}<0.05 \mathrm{vs}$. before treatment; ${ }^{\mathrm{b}} \mathrm{P}<0.05 \mathrm{vs}$. control group in the same period. $\mathrm{NO}$, nitric oxide; ET-1, endothelin-1; ICAM-1, intercellular adhesion molecule-1.

Table VIII. Examination results of CMT, RNV and BCVA.

\begin{tabular}{|c|c|c|c|}
\hline Groups & CMT $(\mu \mathrm{m})$ & $\operatorname{RNV}\left(\mathrm{mm}^{2}\right)$ & BCVA \\
\hline \multicolumn{4}{|l|}{ Control } \\
\hline Before treatment & $442.4 \pm 4.7$ & $26.7 \pm 1.2$ & $0.42 \pm 0.04$ \\
\hline 1 month after treatment & $355.1 \pm 5.8^{\mathrm{a}}$ & $20.1 \pm 1.6^{\mathrm{a}}$ & $0.55 \pm 0.08^{\mathrm{a}}$ \\
\hline 3 months after treatment & $267.8 \pm 5.1^{\mathrm{a}}$ & $12.5 \pm 1.7^{\mathrm{a}}$ & $0.65 \pm 0.07^{\mathrm{a}}$ \\
\hline \multicolumn{4}{|l|}{ Observation } \\
\hline Before treatment & $445.9 \pm 4.3$ & $26.9 \pm 1.4$ & $0.41 \pm 0.06$ \\
\hline 1 month after treatment & $320.5 \pm 3.8^{\mathrm{a}, \mathrm{b}}$ & $13.1 \pm 1.5^{\mathrm{a}, \mathrm{b}}$ & $0.70 \pm 0.03^{\mathrm{a}, \mathrm{b}}$ \\
\hline 3 months after treatment & $220.1 \pm 2.8^{\mathrm{a}, \mathrm{b}}$ & $5.2 \pm 1.1^{\mathrm{a}, \mathrm{b}}$ & $0.81 \pm 0.06^{\mathrm{a}, \mathrm{b}}$ \\
\hline
\end{tabular}

CMT and RNV levels are decreased significantly and BCVA level is increased remarkably after treatment. The amplitude of variation at 3 months after treatment is more significant than that at 1 month after treatment, and it is also more obvious in the observation group than that in the control group $(\mathrm{P}<0.05)$. ${ }^{\mathrm{P}}<0.05$ vs. before treatment; ${ }^{\mathrm{b}} \mathrm{P}<0.05$ vs. control group in the same period. CMT, central macular thickness; $\mathrm{RNV}$, retinal neovascularization; BCVA, best corrected visual acuity.

decreased compared with those in the control group, indicating that the hyperlipemia indexes in DR patients will be evidently improved after treatment with TA combined with AG. Research has shown that inflammation exerts effects in the occurrence and development of DR. As the inflammatory cells increase, the clinopathological changes of DR include increased inflammatory cytokines and oxidative stress damage (19). This study revealed that after treatment, the levels of IL-6, MPO and TNF- $\alpha$ in the two groups of patients were remarkably lower than those before treatment, and those in the observation group were significantly lower than those in the control group. After treatment, the levels of MDA and CAT in the observation group were markedly reduced, while the SOD level was obviously increased, and the variation amplitude in the observation group was more significant than that in the control group. The combination of TA and AG in the treatment of DR can prevent excessive production of inflammatory cytokines to cause irreversible oxidative stress damage to cells, similarly to previously reported (20).
VEGF is a highly conserved glycoprotein, a powerful vascular permeability factor, an endothelial cell-specific mitogen and an angiogenic factor, which plays a pivotal role in physiological and pathological angiogenesis as well as the etiology of DR. VEGF stimulates and facilitates vascular permeability, vasodilation dependent on endothelium, monocyte chemotaxis and tissue factor production, and they can lead to microvascular complications $(21,22)$. It was discovered in this study that before treatment, there was no significant difference in the VEGF level between the two groups. After treatment, the VEGF level in the two groups of patients was notably lower than that before treatment, and it was markedly lower in the observation group than that in the control group. Some studies have indicated that inhibition of CMT and RNV after laser treatment can effectively improve retinal thickness and neovascular leakage. Other studies have reported that TA therapy notably improves retinopathy and vision $(23,24)$. In this study, no significant differences in the levels of CMT, RNV and BCVA were found between the two 
groups of patients before treatment. After treatment, CMT and RNV levels in the observation group declined prominently, while the BCVA level was raised significantly, and the variation amplitude in the observation group was larger than that in the control group. Some scholars consider that ET-1 has abnormal changes in DR in the early stage. Non-specific NOS inhibitors can protect the retina from ischemic damage, denoting that $\mathrm{NO}$ exerts a pivotal effect in the pathogenesis of DR. ICAM-1 also has certain clinical reference value in DR $(25,26)$. This study revealed that before treatment, there were no significant differences in the levels of NO, ET-1 and ICAM-1 between the two groups of patients. After treatment, the levels of NO, ET-1 and ICAM-1 in the two groups of patients were obviously lower than those before treatment, and those in the observation group were evidently lower than those in the control group, which are similar to the above study results.

In conclusion, DR patients were enrolled and treated. The therapeutic effect and adverse reactions were observed, the changes in serum inflammatory factors and oxidative stress factors before treatment were detected, the level of serum VEGF was measured, CMT, RNV and BCVA were observed, the effects of TA combined with AG on vascular endothelial function and retinal function of DR patients were examined, to confirm that TA combined with AG inhibited inflammation and oxidative stress and improved vascular endothelial function and retinal function in DR patients. The overall effect of the combined therapy is good, and its application is worthy of popularizing.

\section{Acknowledgements}

Not applicable.

\section{Funding}

No funding was received.

\section{Availability of data and materials}

The data generated or analyzed during this study are included in this published article.

\section{Authors' contributions}

$\mathrm{KX}$ and MZ designed the study and performed the experiments, KX and HQ collected the data, MZ and HQ analyzed the data, KX and MZ prepared the manuscript. All the authors read and approved the final manuscript.

\section{Ethics approval and consent to participate}

This study was approved by the Ethics Committee of the Taizhou Second People's Hospital Affiliated to Yangzhou University (Taizhou, China). Signed informed consents were obtained from the patients and/or guardians.

\section{Patients consent for publication}

Not applicable.

\section{Competing interests}

The authors declare that they have no competing interests.

\section{References}

1. Zhang HY, Ruan LB, Li Y, Yang TR, Liu WJ, Jiang YX, Li TR, Quan J and Xuan W: ICOS/ICOSL upregulation mediates inflammatory response and endothelial dysfunction in type 2 diabetes mellitus. Eur Rev Med Pharmacol Sci 22: 8898-8908, 2018.

2. Sharma S, Oliver-Fernandez A, Liu W, Buchholz P and Walt J: The impact of diabetic retinopathy on health-related quality of life. Curr Opin Ophthalmol 16: 155-159, 2005.

3. Zheng Y, He M and Congdon N: The worldwide epidemic of diabetic retinopathy. Indian J Ophthalmol 60: 428-431, 2012.

4. Fletcher EL, Phipps JA, Ward MM, Puthussery T and Wilkinson-Berka JL: Neuronal and glial cell abnormality as predictors of progression of diabetic retinopathy. Curr Pharm Des 13: 2699-2712, 2007.

5. Barber AJ: A new view of diabetic retinopathy: A neurodegenerative disease of the eye. Prog Neuropsychopharmacol Biol Psychiatry 27: 283-290, 2003.

6. Antonetti DA, Barber AJ, Bronson SK, Freeman WM, Gardner TW, Jefferson LS, Kester M, Kimball SR, Krady JK, LaNoue KF, et al; JDRF Diabetic Retinopathy Center Group: Diabetic retinopathy: Seeing beyond glucose-induced microvascular disease. Diabetes 55: 2401-2411, 2006.

7. Lieth E, Gardner TW, Barber AJ and Antonetti DA; Penn State Retina Research Group: Retinal neurodegeneration: Early pathology in diabetes. Clin Exp Ophthalmol 28: 3-8, 2000.

8. Pemp B, Garhofer G, Weigert G, Karl K, Resch H, Wolzt M and Schmetterer L: Reduced retinal vessel response to flicker stimulation but not to exogenous nitric oxide in type 1 diabetes. Invest Ophthalmol Vis Sci 50: 4029-4032, 2009.

9. Luo D, Fan Y and Xu X: The effects of aminoguanidine on retinopathy in STZ-induced diabetic rats. Bioorg Med Chem Lett 22: 4386-4390, 2012.

10. Mahmood D, Singh BK and Akhtar M: Diabetic neuropathy: Therapies on the horizon. J Pharm Pharmacol 61: 1137-1145, 2009.

11. Joussen AM, Doehmen S, Le ML, Koizumi K, Radetzky S, Krohne TU, Poulaki V, Semkova I and Kociok N: TNF-alpha mediated apoptosis plays an important role in the development of early diabetic retinopathy and long-term histopathological alterations. Mol Vis 15: 1418-1428, 2009.

12. Noor JI, Ikeda T, Mishima K, Aoo N, Ohta S, Egashira N, Iwasaki K, Fujiwara M and Ikenoue T: Short-term administration of a new free radical scavenger, edaravone, is more effective than its long-term administration for the treatment of neonatal hypoxic-ischemic encephalopathy. Stroke 36: 2468-2474, 2005.

13. Bolton WK, Cattran DC, Williams ME, Adler SG, Appel GB, Cartwright K, Foiles PG, Freedman BI, Raskin P, Ratner RE, et al; ACTION I Investigator Group: Randomized trial of an inhibitor of formation of advanced glycation end products in diabetic nephropathy. Am J Nephrol 24: 32-40, 2004.

14. Eldred WD and Blute TA: Imaging of nitric oxide in the retina. Vision Res 45: 3469-3486, 2005.

15. Jonas JB, Kreissig I, Söfker A and Degenring RF: Intravitreal injection of triamcinolone for diffuse diabetic macular edema. Arch Ophthalmol 121: 57-61, 2003.

16. Jakus V and Rietbrock N: Advanced glycation end-products and the progress of diabetic vascular complications. Physiol Res 53: 131-142, 2004

17. Qazi Y, Maddula S and Ambati BK: Mediators of ocular angiogenesis. J Genet 88: 495-515, 2009.

18. Li ZP, Xu X, Huang YF, Zhu JF, Wang XJ, Hu HH and He ZP: Exogenous advanced glycosylation end products induce diabetes-like vascular dysfunction in normal rats: A factor for occurrence of diabetic retinopathy. Zhonghua Yan Ke Za Zhi 39: 352-356, 2003 (In Chinese).

19. Yin Y, Chen F, Wang W, Wang $\mathrm{H}$ and Zhang X: Resolvin D1 inhibits inflammatory response in STZ-induced diabetic retinopathy rats: Possible involvement of NLRP3 inflammasome and NF- $\kappa$ B signaling pathway. Mol Vis 23: 242-250, 2017.

20. Mishra A and Newman EA: Aminoguanidine reverses the loss of functional hyperemia in a rat model of diabetic retinopathy. Front Neuroenergetics 3: 10, 2012. 
21. Sun W, Yu WY, Yu DJ, Zhao TL, Wu LJ and Han WY: The effects of recombinant human growth hormone $(\mathrm{rHGH})$ on survival of slender narrow pedicle flap and expressions of vascular endothelial growth factor (VEGF) and classification determinant 34 (CD34). Eur Rev Med Pharmacol Sci 22: 771-777, 2018.

22. Lenz T, Haak T, Malek J, Gröne HJ, Geiger H and Gossmann J: Vascular endothelial growth factor in diabetic nephropathy. Kidney Blood Press Res 26: 338-343, 2003.

23. Mehta H, Gillies MC and Fraser-Bell S: Combination of vascular endothelial growth factor inhibitors and laser therapy for diabetic macular oedema: A review. Clin Exp Ophthalmol 44: 335-339, 2016.

24. Riches K, Franklin L, Maqbool A, Peckham M, Adams M, Bond J, Warburton P, Feric NT, Koschinsky ML, O'Regan DJ, et al: Apolipoprotein(a) acts as a chemorepellent to human vascular smooth muscle cells via integrin $\alpha \mathrm{V} \beta 3$ and RhoA/ROCK- mediated mechanisms. Int J Biochem Cell Biol 45: 1776-1783, 2013.
25. Arden GB and Sivaprasad S: The pathogenesis of early retinal changes of diabetic retinopathy. Doc Ophthalmol 124: 15-26, 2012.

26. Giove TJ, Deshpande MM, Gagen CS and Eldred WD: Increased neuronal nitric oxide synthase activity in retinal neurons in early diabetic retinopathy. Mol Vis 15: 2249-2258, 2009.

This work is licensed under a Creative Commons Attribution-NonCommercial-NoDerivatives 4.0 International (CC BY-NC-ND 4.0) License. 